\title{
Approximate analytical solution of the integro-differential model of bulk crystallization in a metastable liquid with mass supply (heat dissipation) and crystal withdrawal mechanism
}

\author{
Irina Nizovtseva ${ }^{1}$, Alexandr Ivanov ${ }^{2}$, and Irina Alexandrova ${ }^{2}$ \\ ${ }^{1}$ Friedrich-Schiller-Universitat Jena \\ ${ }^{2}$ Ural Federal University named after the first President of Russia B N Yeltsin
}

March 5, 2021

\begin{abstract}
This paper is devoted to an approximate analytical solution of an integro-differential model describing the process of nucleation and growth of particles in crystallizers, taking into account the thermal-mass exchange with the environment and the removal of product crystals from the metastable medium. The method developed in this work for solving model equations (kinetic equation for the particle size distribution function and balance equations for temperature/impurity concentration) is based on using the saddle point method for calculating the Laplace-type integral. It is shown that the degree of metastability of the liquid decreases with time at a fixed value of the mass inflow from the outside (heat flow to the outside). The crystal size distribution function has the form of an irregular bell-shaped curve, which increases with the intensification of heat and mass exchange with the environment.
\end{abstract}

\section{Hosted file}

crystal_growth_sent.pdf available at https://authorea.com/users/399832/articles/ 512244-approximate-analytical-solution-of-the-integro-differential-model-of-bulkcrystallization-in-a-metastable-liquid-with-mass-supply-heat-dissipation-and-crystalwithdrawal-mechanism 

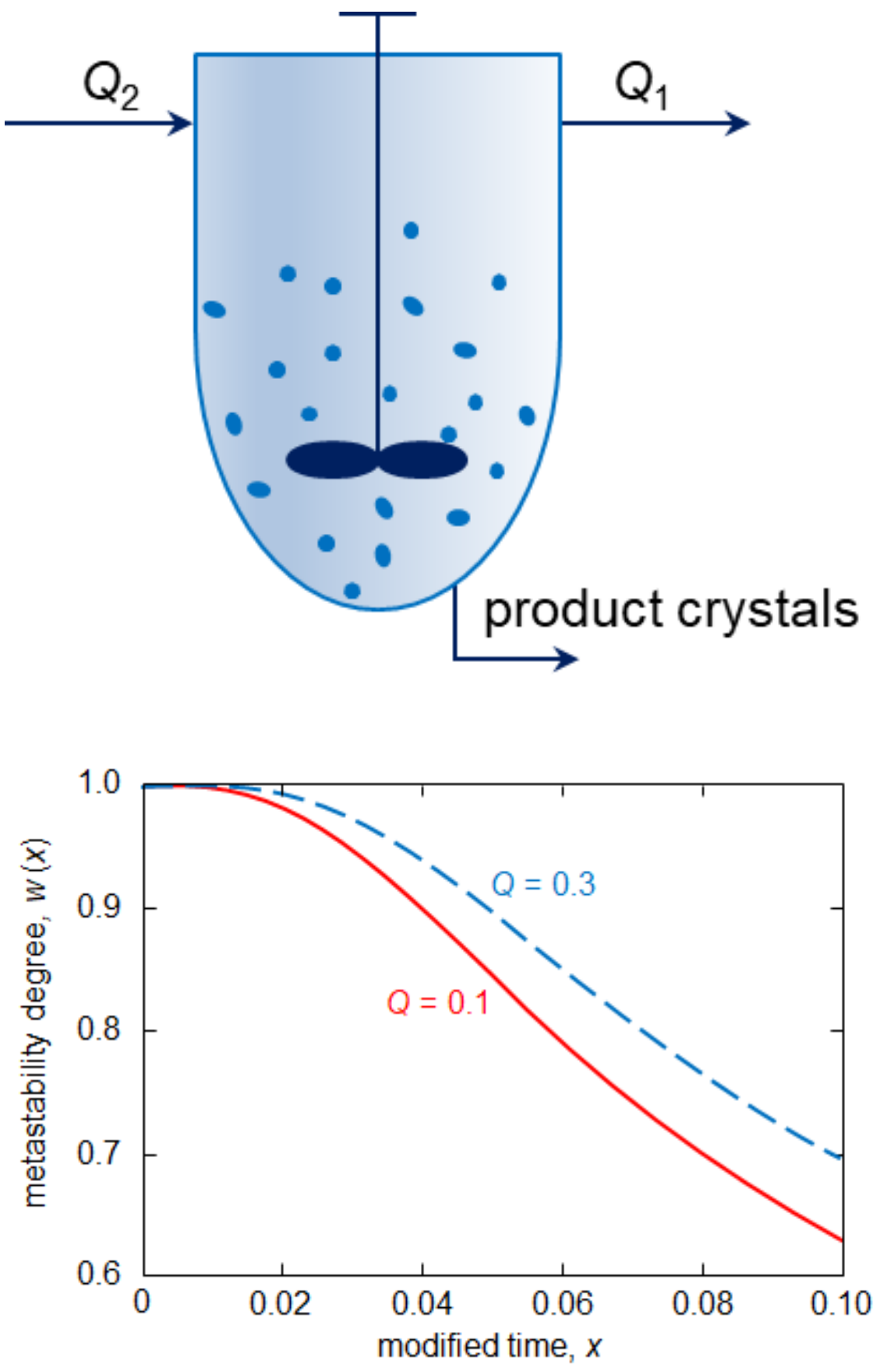


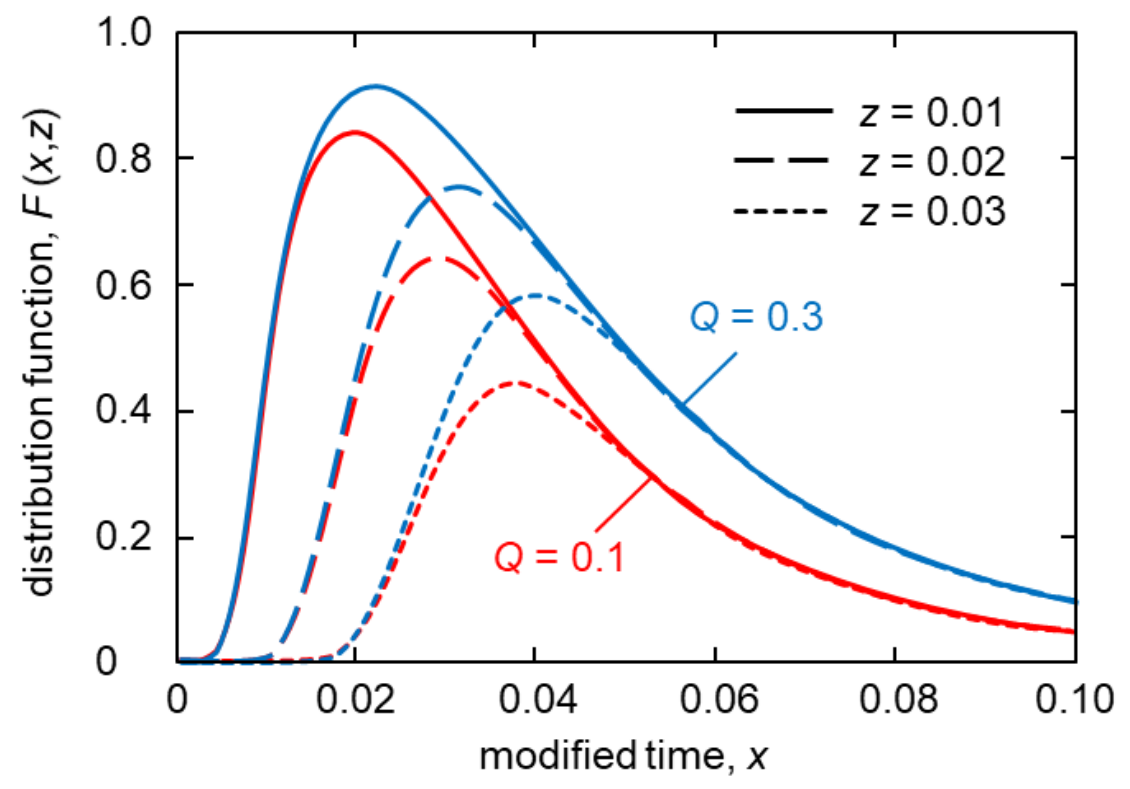

